\title{
Strumenti di gestione dei costi e misure di performance negli attuali contesti competitivi
}

\author{
Lino Cinquini ${ }^{*}$ Rosa Alba Miraglia**, Riccardo Giannetti ${ }^{* * *}$
}

\begin{abstract}
Cost management tools and performance measurement systems in the new competitive context

The topic of cost management tools and performance measurement systems has been addressed in the field of management studies with the aim of identifying the factors that have caused and that determine the success of companies in global competition.

It is believed that, today and in the next future, the key dimensions of competitive advantage of companies are the following: sustainability, intellectual capital and intercompany integration. These dimensions, having a common matrix represented by the complexity, must be integrated in the formulation of the business strategies. This requires the development of suitable cost management tools and performance measures to verify the achievement of the strategies.

But what are the links that are created between strategy, in its various manifestations, and cost management tools and performance measurement? This special issue of the Journal is intended to analyze such links.
\end{abstract}

Keywords: environmental management, sustainability, environmental accounting, cost management, performance measurement.

* Scuola Superiore Sant'Anna di Pisa, lino.cinquini@sssup.it.

** Università degli Studi di Catania, Dipartimento di Economia e Impresa, miraglia@unict.it.

*** Università di Pisa, Dipartimento di Economia e Management, riccardo.giannetti@unipi.it.

Management Control 2, 2016 - Special Issue 


\section{Rapporto tra dimensioni chiave per il vantaggio competitivo e stru- menti di gestione dei costi-sistema di performance}

Il tema del cambiamento degli strumenti di gestione dei costi e dei sistemi di misurazione delle performance (SMP) è stato affrontato nel campo degli studi di management accounting con l'obiettivo di individuare i fattori che lo hanno causato e che determinano il successo delle aziende nella competizione globale.

Si ritiene che, oggi e nel prossimo futuro, le dimensioni chiave per il vantaggio competitivo da parte delle imprese siano le seguenti: sostenibilità, capitale intellettuale e integrazione interaziendale, di cui in estrema sintesi si riportano gli aspetti di fondo.

La sostenibilità richiama la gestione responsabile del business nella sua triplice accezione economica, sociale ed ambientale.

Il capitale intellettuale identifica invece le tre fondamentali aree di generazione dei beni intangibili: la capacità di creare innovazioni, lo sviluppo di modalità organizzative originali del business e del sistema delle relazioni e, infine, l'accumulazione di competenze nel capitale umano aziendale.

L'integrazione interaziendale, nella forma di collegamenti tra imprese, aumenta infine le possibilità di riconfigurazione del modello di business, di condivisione di risorse e di integrazione di capacità e competenze.

Le dimensioni chiave appena accennate, pur essendo eterogenee e interrelate tra di loro, hanno una comune matrice rappresentata dalla complessità, cioè da un attributo dell'ambiente. Con il termine complessità si indica la misura con cui un'azienda fa fronte a molteplici, diversi e interdipendenti ambienti ${ }^{1}$. Nell'ambiente propriamente complesso, come quello attuale, $\mathrm{i}$ fattori che i soggetti decisionali devono considerare sono pertanto numerosi e/o dissimili.

La consapevolezza dell'importanza delle dimensioni chiave prima citate porta l'impresa ad integrarle nella formulazione della propria strategia. Ciò richiede lo sviluppo di idonei strumenti di gestione dei costi e misure di performance per misurare e verificare il raggiungimento degli obiettivi di tali strategie.

Si pone allora la necessità di determinare il tipo di legame (mono o bidirezionale) che si crea tra strategia, nelle sue diverse manifestazioni, e strumenti di gestione dei costi e SMP.

${ }^{1}$ Il campo di variabilità della complessità va da un minimo (ambiente semplice) ad un massimo (ambiente propriamente complesso). 
Come si osserva nella maggior parte della letteratura sul tema, il legame è bidirezionale (Williamson et al., 2006; Miraglia, 2012; Goyal et al., 2013). Infatti, da un lato, la strategia influenza specifici strumenti di gestione dei costi e indicatori di performance, nei quali essa viene tradotta; dall'altro lato, tali strumenti ed indicatori di performance supportano i manager nel processo decisionale relativo all'implementazione della stessa strategia. La fig. n. 1 riporta quanto appena espresso.

Figura 1 - Legami tra le strategie collegate con le dimensioni chiave per il vantaggio competitivo e strumenti di gestione dei costi e SMP

\begin{tabular}{|l|l|}
\hline $\begin{array}{l}\text { Strategie collegate alle dimensioni chiave } \\
\text { per il vantaggio competitivo }\end{array}$ \\
\hline - sostenibilità \\
- capitale intellettuale \\
- integrazione interorganizzativa
\end{tabular}

Il legame appena riportato non è il solo tema emergente nella letteratura più recente sugli strumenti di gestione dei costi e sui sistemi di misurazione. Ad esso si aggiungono infatti i nuovi problemi che derivano dai caratteri emergenti della competitività: l'identificazione dei drivers della performance della sostenibilità e del loro impatto sui diversi stakeholders (Epstein \& Roy, 2001; Booker et al., 2007; Lisi, 2015); le modalità con cui indicatori chiave di performance (KPI) relativi alla sostenibilità vengono utilizzati a supporto delle decisioni, della pianificazione e della misurazione delle performance aziendali (Gond et al., 2012; Marelli, 2015); i costi e le performance delle attività svolte per sviluppare nuovi prodotti e servizi (Davila e Wouters, 2004; Booker et al., 2007; Chiesa e Frattini, 2009; Moll, 2015.); la crescita dell'importanza delle informazioni collegate ai beni intangibili (Wyatt, 2008; Ittner, 2008; Cinquini et al., 2012); l'efficacia dei sistemi per il controllo e la performance di nuove forme organizzative ibride (inter-organizational networks) (Cooper e Slagmulder, 2004; Miraglia, 2005; Coad e Cullen, 2006; Agndal e Nilsson, 2009; Miraglia e Leotta, 2010; Mancini, 2011; Marchi, 2011); ecc.

Sono anche effettuati studi e proposte innovative legati a: l'analisi dei costi ambientali (Bartolomeo, 1997; Pisani, 2002), il costo del ciclo di vita del prodotto (Kloepffer, 2008), l'analisi dei processi in logica di relazioni interaziendali (Basu, 2001; Beamon e Chen, 2001; Chab e Qi, 2003).

Risultano invece ancora non particolarmente approfonditi: 
- l'apprezzamento del reale grado di diffusione, conoscenza ed utilizzo nelle imprese di strumenti ed approcci strategici di misurazione dei costi e delle performance che inseriscano in modo efficace le dimensioni chiave suindicate nell'ambito dei sistemi informativi e di controllo, per il supporto alle decisioni strategiche ed operative della gestione;

- gli aspetti legati all'impatto della presenza e utilizzo di strumenti di cost e performance management sullo sviluppo di nuovi prodotti-servizi sostenibili e sull'accumulo di risorse intangibili;

- l'esplorazione della dimensione dei sistemi di misurazione in contesti interorganizzativi.

Si evidenzia pertanto la necessità di studiare, sul piano teorico ed empirico, le tendenze in atto nei supporti informativo-contabili per la gestione dei costi e delle performance considerando in particolare la sostenibilità, il capitale intellettuale e l'integrazione interaziendale quali componenti determinanti per la competitività delle imprese.

\section{Tendenze evolutive degli strumenti di gestione dei costi e del SMP nei nuovi ambienti socio-economici}

I contributi raccolti in questo numero di Management Control sono stati originariamente presentati nel Workshop "Misure di performance e sistemi di informazione e controllo nei nuovi ambienti socio-economici" tenutosi a Catania il 22-23 ottobre 2015. L'organizzazione di tale evento ha avuto come motivazione principale la presentazione di alcuni risultati del progetto di ricerca nazionale (PRIN 2008) dal titolo "Gestione strategica dei costi e delle performance per le sfide della competitività e della sostenibilità".

Questo progetto nazionale si è articolato in tre unità operative locali, ciascuna con un proprio segmento di ricerca, ben individuato e interconnesso con quello delle altre. In particolare:

- l'unità di ricerca I della Scuola Superiore Sant'Anna di Pisa (Responsabile nazionale e dell'unità Lino Cinquini) si è occupata della gestione strategica dei costi e delle performance per il vantaggio competitivo sostenibile;

- l'unità di ricerca II dell'Università di Pisa (Responsabile Riccardo Giannetti) si è occupata della gestione strategica dei costi e delle performance nello sviluppo di nuovi prodotti-servizi;

- l'unità di ricerca III dell'Università di Catania (Responsabile Rosa Alba Miraglia) si è occupata delle misure di performance in contesti di cooperazione interaziendale. 
La ricerca si è rivolta pertanto verso l'analisi dell'evoluzione dei sistemi di costing e di misurazione delle performance utilizzati per il supporto alle decisioni strategiche e per il controllo di gestione a livello d'azienda/business unit.

I lavori pubblicati in questo volume costituiscono le relazioni presentate nel Workshop direttamente collegate al PRIN indicato, ed alcuni paper selezionati tra quelli presentati nello stesso Workshop, che ha rappresentato un momento di riflessione di elevato valore scientifico su questi temi.

Il problema di ricerca che lega tutti i contributi è l'impatto che la complessità ambientale ha sugli strumenti di gestione dei costi e sul sistema di misurazione delle performance (SMP). In particolare, essi mettono in evidenza la criticità del rapporto tra le dimensioni chiave per il vantaggio competitivo dell'azienda, gli strumenti di gestione dei costi e le misure di performance. In queste pagine si chiariscono innanzitutto quali nuove dimensioni si ritengono fondamentali per il vantaggio competitivo dell'azienda; si esamina quindi il rapporto tra tali dimensioni, gli strumenti di gestione dei costi e il SMP; si individuano infine le tendenze evolutive degli strumenti di gestione dei costi e del SMP che emergono dai contributi pubblicati in questo numero.

La relazione di Lino Cinquini, Emilio Passetti e Andrea Tenucci, dal titolo "La sostenibilità ambientale in azienda: quale relazione tra disclosure volontaria e gestione interna?", analizza il legame esistente tra comunicazione volontaria ambientale e gestione ambientale interna al fine di capire se e come le due dimensioni sono associate e coerenti tra loro. Il tema, non particolarmente approfondito in letteratura, è osservato dal punto di vista teorico ed empirico. Con il primo, gli autori individuano, in linea con precedenti studi, quattro categorie rappresentative di possibili associazioni, avvalendosi di una matrice alto-basso che rappresenta le due dimensioni indicate. L'analisi empirica si avvale invece di un campione di aziende operanti in settori considerati ad alto impatto ambientale. La ricerca fa uso di un questionario per quanto riguarda la dimensione della gestione interna mentre calcola un indice di disclosure per ciò che attiene la dimensione esterna. I risultati mostrano una prevalenza della gestione ambientale interna rispetto alle pratiche di comunicazione volontaria. L'associazione tra le due dimensioni risulta presente solo per un sottoinsieme di aziende, che sono anche le più attente alla sostenibilità ambientale.

Il lavoro offre alla letteratura due contributi principali. Il primo è l'analisi empirica dell'associazione tra dimensione interna ed esterna finalizzata a comprendere il grado di penetrazione della sostenibilità ambientale nei meccanismi decisionali e di comunicazione esterna dell'azienda. Il se- 
condo contributo, di natura teorica, mette in evidenza l'esigenza di stimolare future ricerche per approfondire l'associazione e la coerenza tra le due dimensioni collegando il tema ad aspetti di cambiamento organizzativo.

La relazione di Riccardo Giannetti e Alessandro Marelli, dal titolo "Il ruolo degli strumenti di cost management nello sviluppo di nuovi prodotti sostenibili", esamina invece l'impiego degli strumenti di cost management nel processo di sviluppo di nuovi prodotti (SNP). Il tema è osservato dal punto di vista teorico ed empirico. Con il primo gli autori elaborano un modello nel quale l'adozione nello SNP di vari strumenti (Activity-Based Costing/Management, Target Costing e Design to Cost, Life Cycle Costing e Total Cost of Ownership) è studiato esaminando alcune variabili (o fattori) di contesto. Con il secondo, la distribuzione di un questionario ad un campione di aziende consente di osservare sia il grado di adozione dei vari strumenti prima esaminati nel contesto italiano, sia l'associazione tra il loro utilizzo e le variabili di contesto considerate.

Lo studio contribuisce allo sviluppo della conoscenza sulle possibili influenze che le variabili di contesto possono avere nell'adozione in azienda di strumenti di cost management. In particolare dai risultati emerge una significativa associazione tra strategie di sostenibilità e utilizzo di strumenti di cost management a supporto dello SNP.

La relazione di Rosa Alba Miraglia, dal titolo "Modello di controllo e misure di performance nelle relazioni di fornitura tra imprese. Una ricerca empirica nel contesto italiano", ha infine lo scopo di esporre i risultati di un'indagine empirica che cerca di capire se e in quale misura il modello di controllo interorganizzativo più diffuso in Italia influenza le relative misure di performance. Il conseguimento di questo obiettivo ha, innanzitutto, richiesto la ricostruzione della specifica letteratura sui modelli di controllo interorganizzativo per impiegarli, poi, come chiave di lettura dei meccanismi di controllo nelle relazioni di fornitura osservate attraverso una survey condotta nel contesto italiano.

Il contributo offerto è prevalentemente di tipo empirico. Esso individua $i$ caratteri del modello di controllo interorganizzativo maggiormente adottato in Italia e i connessi meccanismi di controllo (prezzi, regole, fiducia), che svolgono la funzione di coordinare gli interessi dei partner; l'influenza che tale modello di controllo esercita sulle relative misure di performance; l'effetto prodotto dai fattori immateriali sui caratteri delle misure.

I paper selezionati si collegano alle dimensioni chiave per il vantaggio competitivo dell'azienda osservate nelle relazioni. L'ordine di pubblicazione di tali paper rispecchia quello adottato per le relazioni. Una eccezione va 
fatta per il primo paper che, esaminando la complessità ambientale, studia quella che precedentemente abbiamo definito matrice comune delle dimensioni chiave e come tale precede gli altri paper, che invece si occupano di specifiche dimensioni.

Il contributo di Eugenio Comuzzi e Filippo Zanin, dal titolo "Dal controllo dell'impresa al controllo della complessità", si propone di capire se ed in quale misura il riferimento al concetto della complessità, che include l'incertezza del contesto esterno e la percezione soggettiva della realtà, è in grado sia di dare una visione organica delle caratteristiche del contesto di riferimento per l'attività di controllo sia di fornire nuove chiavi interpretative sul controllo. Per perseguire questi obiettivi, gli autori delineano uno schema metodologico di riferimento per il controllo in contesti complessi, che consente di osservare i possibili effetti sulla misurazione e il controllo a livello d'azienda. Quindi, presentano approfondimenti relativi ad alcune variabili specifiche del controllo riportando evidenze empiriche tratte dallo studio di un caso aziendale.

Il lavoro offre una chiave metodologica che si propone di sistematizzare, entro un unico concetto, quello della complessità, i fattori capaci di influenzare il disegno e lo svolgimento dell'attività di controllo in azienda.

Il contributo di Stefano Garzella, Daniela Mancini, Raffaele Fiorentino e Rita Lamboglia, dal titolo "Strategie di sostenibilità: motivazione e sistemi di misurazione della performance", analizza le principali sfide che i SMP sono chiamati ad affrontare in seguito all'adozione di strategie di sostenibilità. Il tema è affrontato prima in chiave teorica con l'elaborazione di un framework in grado di esaminare l'impatto potenziale delle strategie di sostenibilità sui SMP. Il modello è sviluppato in base ad un approccio "strategy-action-performance". Successivamente, in base a tale framework si effettua un'analisi empirica, utilizzando la metodologia dello studio di un caso, condotto mediante un'analisi documentale e la realizzazione di interviste.

Il lavoro contribuisce ad arricchire la letteratura sulla relazione tra strategie di sostenibilità e SMP, esaminando le tipologie di cambiamenti che si verificano nei SMP quando le aziende perseguono strategie sociali. Dal punto di vista pratico può essere un punto di riferimento per i manager che intendono sviluppare nelle loro aziende strategie di sostenibilità e valutare il loro impatto sia attraverso misure di performance economico-finanziario sia con indicatori di carattere sociale ed ambientale.

Il contributo di Anna Arcari, Anna Pistoni, Enrico Moretto, Paola Ossola e Daniele Tonini, dal titolo "The Role of Managerial Control in Innovation Processes: An empirical analysis in Italian firms", ha due obiettivi: il 
primo, testare empiricamente se i SMP hanno un impatto sulle performance dell'innovazione; il secondo, mettere in evidenza le caratteristiche dei SMP che possono favorire lo sviluppo dell'innovazione. Il conseguimento di questi obiettivi ha richiesto, innanzitutto, la ricostruzione della letteratura sulle peculiarità assunte dai SMP nelle aziende innovative; quindi un'indagine empirica su un campione di aziende innovative, alle quali somministrare un questionario.

Lo studio contribuisce alla letteratura sull'osservazione empirica dei caratteri che i SMP dovrebbero possedere nelle aziende innovative, ancora oggi non sufficientemente approfondita e articolata. Questo arricchimento permette anche di trarre considerazioni significative circa il modo in cui è opportuno progettare i SMP a garanzia dell'innovazione.

Il contributo di Antonio Leotta, dal titolo "Inter-firm coordination as an information problem. The case of a divisionalized company", si propone infine di spiegare le forme ibride di coordinamento inter-organizzativo studiando il coordinamento come problema informativo. Questo obiettivo viene perseguito sia attraverso una concettualizzazione teorica, che presenta il coordinamento come un processo dialettico tra le diverse entità organizzative coinvolte, sia applicando il framework teorico come una lente per evidenziare le dinamiche e i fabbisogni di coordinamento nelle relazioni tra le business units di una azienda multidivisionale e i sub-fornitori.

Il contributo offerto è sia di tipo teorico che empirico. Sotto l'aspetto teorico, studiare il coordinamento come problema informativo consente di spiegare meglio la formazione di forme ibride di coordinamento. Sotto l'aspetto empirico, il caso-studio offre elementi che arricchiscono lo schema teorico di partenza, evidenziando come le dinamiche di coordinamento inter-organizzativo, tra business units e sub-contractor, siano largamente influenzate dalle dinamiche intra-organizzative, tra le business unit e tra queste e il livello corporate.

\section{Bibliografia}

Agndal H., Nilsson U. (2009), Interorganization cost management in the exchange process, Management Accounting Research, 20, pp. 85-101.

Doi: 10.1016/S0361-3682(03)00020-5.

Bartolomeo M. (1997), La contabilità ambientale d'impresa, Bologna, il Mulino.

Basu R. (2001), New criteria of performance management: a transition from enterprise to collaborative supply chain, Measuring Business Excellence, 5 (4).

Doi: EUM0000000006514. 
Beamon B.M., Chen V. (2001), Performance analysis of conjoined supply chain, International Journal of Production Research, Vol. 39, No. 17.

Booker D.M., Drake R.A., Heitger D.L. (2007), New Product Development: How Cost Information Precision Affects Designer Focus and Behavior in a Multiple Objective Setting, Behavioral Research in Accounting 19, 19-41.

Doi: 10.2308/bria.2007.19.1.19.

Chan F., Qi H.J. (2003), Feasibility of performance measurement system for supply chain: a process-based approach and measures, Integrated Manufacturing System, Vol. 14, No 3. Doi: 10.1108/09576060310463145.

Chiesa V., Frattini F. (2009), Evaluation and performance Measurement of Research and Development. Techniques and Perspectives for Multi-Level Analysis, E. Elgar Publishing.

Cinquini L., Passetti E., Tenucci A. \& Frey M. (2012), Analyzing intellectual capital information in sustainability reports: some empirical evidence, Journal of Intellectual Capital, 13(4), pp. 531-561.

Coad A.F., Cullen J. (2006), Inter-organisational cost management: Towards an evolutionary perspective, Management Accounting Research, 17, pp. 342-369.

Doi: 10.1016/j.mar.2006.02.003.

Cooper R., Slagmulder R. (2004), Interorganizational cost management and relational context, Accounting, organizations and Society, 29, pp. 1-26.

Doi: 10.1016/S0361-3682(03)00020-5.

Davila. T., Wouters M. (2004), Designing Cost-Competitive Technology Products through Cost Management, Accounting Horizons, 18(1), pp. 13-26.

Doi: 10.2308/acch.2004.18.1.13.

Epstein M.J. \& Roy M.-J. (2001), Sustainability in Action: Identifying and Measuring the Key Performance Drivers, Long Range Planning, 34(5), pp. 585-604.

Doi: 10.1016/S0024-6301(01)00084-X.

Gond J.-P., Grubnic S., Herzig C. \& Moon J. (2012), Configuring management control systems: Theorizing the integration of strategy and sustainability, Management Accounting Research 23(3), pp. 205-223. Doi: 10.1016/j.mar.2012.06.003.

Goyal P., Rahman Z., Kazmi A. (2013), Corporate sustainability performance and firm performance research: literature review and future research agenda, Management Decision, pp. 51(2), 361-379.

Ittner C.D. (2008), Does measuring intangibles for management purposes improve performance? A review of the evidence, Accounting \& Business Research 38(3), pp. 261-272.

Kloepffer W. (2008), Life cycle sustainability assessment of products, The International Journal of Life Cycle Assessment, 13(2), pp. 89-95. Doi: 10.1065/lca2008.02.376.

Lisi I.E. (2015), Translating environmental motivations into performance: The role of environmental performance measurement systems, Management Accounting Research, 29, pp. 27-44. Doi: 10.1016/j.mar.2015.06.001.

Mancini D. (2011), L'azienda-rete e le decisioni di partnership: il ruolo del sistema informativo relazionale, Management Control, 1, pp. 65-97. Doi: 10.3280/MACO2011-001004.

Marchi L. (2011), L'evoluzione del controllo di gestione nella prospettiva informativa e gestionale esterna, Management Control, 3, pp. 5-16. Doi: 10.3280/MACO2011-003001.

Marelli, A. (2015). The evolving role of environmental management accounting in internal decision-making: a research note, International Journal of Accounting, Auditing and Performance Evaluation 11(1), pp. 14-47. Doi: 10.1504/IJAAPE.2015.068081.

Miraglia R.A. (2005), Reti di aziende e sistema informativo interorganizzativo, G. Giappichelli. 
Miraglia R.A., Leotta A. (2010), The interaction between information and trust in the control of transactional relationship: theoretical perspectives and empirical support. In Epstein M., Manzoni J.F., Davila A., Performance Measurement \& Management Control Innovative Concepts \& Practices, vol. 20, Emerald Group Publishing, Ltd, pp. 143-175.

Miraglia R.A. (2012), Nuove tendenze nei sistemi di controllo e di misurazione delle performance, Management Control, 2, pp. 5-14. Doi: 10.3280/MACO2012-002001.

Moll J. (2015), Editorial: Special issue on innovation and product development, Management Accounting Research, 28, pp. 2-11. Doi: 10.1016/j.mar.2015.05.003.

Pisani M. (2002), L’ambiente naturale nel bilancio di esercizio. Costi e passività ambientali, Milano, FrancoAngeli.

Williamson D., Lynch-Wood G., Ramsay J. (2006), Drivers of environmental behaviour in manufacturing SMEs and the implications for CSR, Journal of Business Ethics, 67(3), pp. 317-330. Doi: 10.1007/s10551-006-9187-1.

Wyatt A. (2008), What financial and non-financial information on intangibles is valuerelevant? A review of the evidence, Accounting \& Business Research 38(3), pp. 217 256. 